R. Sadanandan, J. Fleck, W. Meier, P. Griebel and C. Naumann, 2D mixture fraction measurements in a high pressure and high temperature combustion system using NO tracer-LIF, Applied Physics B 106 (2012) 185-196.

The original publication is available at www.springerlink.com

http://dx.doi.org/10.1007/s00340-011-4655-4 


\title{
2D Mixture Fraction Measurements in a High Pressure and High Temperature Combustion System Using NO Tracer-LIF
}

\author{
R. Sadanandan, J. Fleck ${ }^{\star}$, W. Meier, P. Griebel, C. Naumann \\ Institut für Verbrennungstechnik, Deutsches Zentrum für Luft- und Raumfahrt e.V., Pfaffenwaldring 38-40, 70569 Stutttgart, Germany.
}

Received: date / Revised version: date

\begin{abstract}
Mixture fraction measurements in a jet-in-cross flow configuration at high pressures (15 bar) and temperatures (above $1000 \mathrm{~K}$ ) were performed using planar laser induced fluorescence of nitric oxide (NO-PLIF) as trace species. The goal was the evaluation of this tracer LIF technique for the characterization of the mixing of fuel and hot exhaust gas in the mixing channel. The fuel (natural gas (NG) or $\mathrm{H}_{2} / \mathrm{N}_{2} / \mathrm{NG}$ mixture) along with the tracer were injected into the crossflow of the exhaust gas and PLIF measurements were performed in different planes. In order to relate the measured NO-LIF signal to fuel concentration and mixture fraction, effects of pressure, temperature and species concentration were taken into account. Numerical calculations and spectroscopic simulations that mimic the experimental conditions were performed to identify excitation schemes that give optimum correlations between the NO-LIF signal and the mixture fraction. The measured NO-PLIF images were transformed into mixture fraction plots using the computed correlations. The paper reports on the experimental challenges encountered during the measurements and the steps taken to overcome those difficulties. Examples of mixture fraction distributions are presented and discussed. The paper concludes with a detailed analysis on the accuracy of the measured mixture fraction values.
\end{abstract}

\section{Introduction}

In recent decades laser based diagnostic methods have become an integral part of fundamental as well as applied combustion research owing to its capability for remote, nonintrusive and in-situ measurements $[1,2]$. One of the most important quantities in non-premixed combustion is mixture fraction, a measure of the fraction of the mass present that originated from one of the two mixing streams. Different laser based diagnostic methods have been employed to measure the mixture fraction, for example in a non-reacting flow, from a tag added to the fuel [3] and for reacting flows, Rayleigh scattering from the fuel [4], fluorescence from the fuel [5] and

\footnotetext{
^ Fax: +49-711-6862 578, E-mail: Julia.Fleck@ dlr.de
}

Raman scattering [6,7]. At high pressures and high temperatures many of these methods are not applicable or difficult to perform, due to a variety of constraints like limited optical access, high thermal loads encountered or lack of appropriate species.

Among the different laser based diagnostic methods planar laser induced fluorescence (PLIF) has been frequently used for 2D measurements due to its high spatial and temporal resolution and also due to its high sensitivity and species specificity. LIF of nitric oxide (NO) as trace species have been employed by many research groups for the estimation of fuel concentration and temperature [8-11]. NO as trace species is particularly attractive when compared to others as NO has good thermal stability, well-characterized spectroscopy and strong absorption using readily accessible wavelengths of tunable dye lasers currently available [12]. Moreover, NO has excellent fluorescent characteristics and stability when compared to other fuel markers reported in literature like acetone or acetaldehyde. However, at elevated pressures complications arise due to the collisional broadening and shifting of the NO-LIF spectrum $[13,14]$, which can result in an overall reduction in the signal-to-noise ratio, and also due to interference issues $\left(\mathrm{O}_{2}\right.$ and $\mathrm{CO}_{2}$-LIF) $[15,16]$. Furthermore, quantitative concentration measurements using PLIF is not straight forward as it requires a detailed knowledge of the probed species spectroscopy (NO) and also the chemical boundary conditions (species concentration, pressure and temperature) [17]. This is due to the complex dependency of the LIF signal on the mixture fraction as explained in the following sections. The method reported in this paper is similar to the approach followed in $[18,19]$.

The paper reports investigation performed in a jet in cross flow (JICF) configuration under high pressures (15 bar) and temperatures. JICF finds application in an array of engineering problems, such as understanding pollutant dispersion from power plant exhaust ducts, aerospace propulsion, turbine blade cooling in gas turbines, and reheat combustors [20-22]. In the current research 2D fuel mixture fraction in the mixing section (which is optically accessible) of the combustor is determined based on the PLIF of NO which is 
seeded to the fuel mixture. The aim is to visualize the fuel mixing process by using a tracer (here NO) as representative of the local fuel concentration. Since the NO-LIF signal has a non-linear relationship with the mixture fraction, additional numerical calculations and spectroscopic simulations that mimic the experimental scenario were performed to identify correlations between the LIF-signal and the mixture fraction. The computed correlations were then used to transform the NO-PLIF images into mixture fraction plots. The first part of the paper discusses the experimental methodology along with descriptions about the experimental set-up and the experimental challenges that were encountered. This is followed by discussions about the experimental results and the measurement accuracy. The main goal of this research paper is to demonstrate and discuss the feasibility of employing tracer LIF measurements for 2D mixture fraction measurements under high pressures and high temperatures.

\section{Mixture Fraction Measurements at High Pressures and High Temperatures}

\subsection{Basic Principles}

The determination of fuel mixture fraction at high pressures and temperatures relevant to technical gas turbine operating conditions described in this research paper is based on a combination of LIF of seeded NO, simple numerical calculations, and spectroscopic simulations to identify correlations between the theoretical LIF signal $\left(S_{L I F}\right)$ and the mixture fraction. By assuming the mixing process as adiabatic, the simple numerical calculation delivers a data table containing correlations between the temperature, species concentrations and the mixture fraction - that is, the chemical boundary conditions describing the thermochemical state of the investigated flow. This is used as the input parameter for the spectroscopic simulation of the $S_{L I F}$. Since the correlation between $S_{L I F}$ and mixture fraction depends on the LIF excitation scheme, various spectroscopic simulations involving different LIF excitation schemes are carried out to narrow down on the excitation scheme that delivered sharp correlations. The selected correlation is then used to convert the NOLIF signals into 2D-mixture fraction plots.

\subsection{Experimental Configuration}

2.2.1 Mixing Section and High-Pressure Combustion Test Rig Mixture fraction measurements using $\mathrm{NO}$ as the trace species were performed at an optically accessible mixing section, MS ( $25 \times 25 \mathrm{~mm}^{2}$ in cross section). The experimental configuration is shown schematically in Fig. 1. The fuel was injected in a JICF configuration into hot exhaust gas stream. The MS was installed in the high-pressure combustion test rig (HBK-S) of the Institute of Combustion Technology at DLR, Stuttgart. The test rig pressure vessel is equipped with large quartz windows thereby providing excellent optical access to the mixing section. The test rig and the combustor were equipped with a large number of thermocouples and pressure transducers (both absolute and differential) in order to measure temperatures and pressures at several locations. Emissions and exhaust gas composition were measured with an exhaust gas analysis system and an emission probe at the MS inlet. More information about the high pressure test facility can be found elsewhere [24].

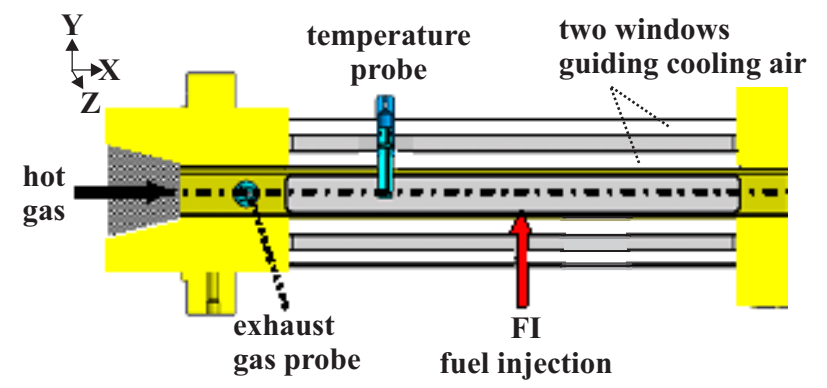

Fig. 1 Schematic of the experimental configuration.

The combustor was operated at a pressure of $15 \mathrm{bar}$ and an exhaust gas $\mathrm{O}_{2}$ concentration of $15 \mathrm{vol} . \%$ at the inlet of MS. The air and the natural gas (NG) fuel flow rates were controlled by high-prescision Corioils flow meters. Mass flow controllers were used to regulate the $\mathrm{H}_{2}$ and $\mathrm{N}_{2}$ flows. The different fuel mixtures that were investigated are listed in Table. 1. In order to achieve sufficient jet penetration into the cross flow, a carrier medium $\left(\mathrm{N}_{2}\right)$ was added to the fuel. Note that in case of F2, the added carrier medium is in addition to the $\mathrm{N}_{2}$ already present in the fuel mixture. The carrier was perfectly mixed to the fuel with the carrier-to-fuel mass flow ratio set at 0.5 for $\mathrm{F} 1$ and 1.0 for $\mathrm{F} 2$, in order to adapt the different $J$ and enable adequate jet penetration into cross flow. So the fuel jet exiting the injector is a mixture of fuel gas and the carrier medium. The corresponding operational parameters (including the carrier medium) are given in Table. 2, where $d$ is the diameter of the fuel injector, $\operatorname{Re}_{j e t}$ is the jet Reynolds number based on $d$ and jet exit velocity, $J$ is the maximum momentum flux ratio between the jet and the cross flow, $\phi_{\text {global }}$ and $f_{\text {global }}$ are the global equivalence ratio and mixture fraction determined from the mass flow of the fuel and the exhaust gases, and $f_{\text {sto }}$ is the stoichiometric mixture fraction.

Table 1 Fuel gas composition in volume fractions.

\begin{tabular}{|l|c|c|c|}
\hline Fuel type & $\mathrm{NG}$ & $\mathrm{H}_{2}$ & $\mathrm{~N}_{2}$ \\
\hline \hline F1 & 100 & 0 & 0 \\
\hline F2 & 4 & 76 & 20 \\
\hline
\end{tabular}

Table 2 Operating Parameters.

\begin{tabular}{|l|c|c|c|c|c|c|}
\hline $\begin{array}{c}\text { Fuel } \\
\text { type }\end{array}$ & $\begin{array}{c}d \\
(\mathrm{~mm})\end{array}$ & $J$ & $\mathrm{Re}_{\text {jet }}$ & $\phi_{\text {global }}$ & $f_{\text {global }}$ & $f_{\text {stoi }}$ \\
\hline F1 & 2.4 & 7.7 & 558000 & 0.47 & 0.029 & 0.059 \\
\hline F2 & 5.6 & 1.7 & 682000 & 0.36 & 0.06 & 0.151 \\
\hline
\end{tabular}

The fuel/carrier temperatures were about $323 \mathrm{~K}$ for $\mathrm{F} 1$ and $313 \mathrm{~K}$ for F2. The bulk exit velocity of the fuel jet was $257 \mathrm{~ms}^{-1}(\mathrm{M}=0.55)$ for $\mathrm{F} 1$ and $199 \mathrm{~ms}^{-1}(\mathrm{M}=0.37)$ for F2. In case of F1, LES simulations have shown that the velocity reduced to less than $130 \mathrm{~ms}^{-1}(\mathrm{M}=0.3)$ within $0.5-1$ jet diameters. So effects of compressibility are not expected in these experiments. The cross flow velocity of the hot exhaust gases (temperature $>1000 \mathrm{~K}$ ) was $\geqslant 150 \mathrm{~ms}^{-1}$. The hot cross flow was generated using a FLOX ${ }^{\circledR}$ combustor 
with extensive internal gas recirculation and operated exclusively with NG. Previous investigations have shown that the combustor produced exhaust gases with uniform temperature distribution and composition [25]. The effects of differential diffusion is assumed negligible for $\mathrm{F} 2$ due to the high $\mathrm{Re}_{\text {jet }}$. For the determination of fuel mixture fraction, initial measurements were performed with $\mathrm{NO}$ in $\mathrm{N}_{2}(25 / 75 \mathrm{vol}$. \%) as the fuel tracer. However, due to strong quenching of the NOLIF signal by the exhaust gases, the tracer composition was changed to $\mathrm{NO}$ in $\mathrm{CO}_{2}(50 / 50 \mathrm{vol}$. \%) (see discussions below). The fuel with the carrier medium was uniformly mixed with $2500 \mathrm{ppm}$ (mass concentration) of the tracer molecule before it was injected into the cross flow.

2.2.2 NO-PLIF System The schematic of the NO-PLIF excitation and detection system is shown in Fig. 2. For the NOPLIF measurements a flashlamp pumped, frequency doubled Nd:YAG laser (Spectra Physics) was used to pump a frequency tripled tunable dye laser (Sirah Precision Scan Dye Laser System, operating on Coumarine 2 dye) at $10 \mathrm{~Hz}$. The UV beam (pulse duration $8 \mathrm{~ns}$ and line width $\approx 0.4 \mathrm{~cm}^{-1}$ ) had a pulse energy of approximately $4 \mathrm{~mJ}$ at $226 \mathrm{~nm}$. The average beam energy at the measurement location was $\approx 2.4 \mathrm{~mJ} /$ pulse due to the reflection losses at the sheet optics and also due to the transmission losses at the pressure vessel windows and the MS windows. The corresponding spectral irradiance was $\approx 4.7 \times 10^{6} \mathrm{~W} / \mathrm{cm}^{2} / \mathrm{cm}^{-1}$ which is below the $5 \times 10^{6} \mathrm{~W} / \mathrm{cm}^{2} / \mathrm{cm}^{-1}$ reported by Namazian et al. [26] for fluorescence saturation in atmospheric flames. The saturation limit increases with increasing pressure and moreover, linearity of the fluroscence signal was verified before the mixture fraction measurements using neutral density (ND) filters. The output was tuned to coincide with the $\mathrm{Q}_{1}(23)$ transition in the $v^{\prime \prime}=0, v^{\prime}=0$ vibrational band of the $A^{2} \Sigma^{+}-X^{2} \Pi$ system of NO molecule. The transition was chosen based on the theoretical mixture fraction-LIF signal correlation presented below. The laser beam was expanded into a sheet of approximately $40 \mathrm{~mm}$ height and $0.4 \mathrm{~mm}$ thickness by means of cylindrical lenses. The sheet optics were mounted in 3D translation tables which enabled measurements at different axial positions and also at planes parallel to the geometrical axes. Subsequent NO fluorescence in the $A-X(0,1)-(0,5)$ bands at 237, 247, 259, 272 and $285 \mathrm{~nm}$ was detected using a combination of UG5 filter and high-reflecting mirror (HR226/0 deg.) which also blocked direct laser scattering at $226 \mathrm{~nm}$.

The NO-PLIF detection system consisted of an imageintensified CCD camera (LaVision Imager Intense with I/I IRO, $1376 \times 1040$ pixels) with achromatic UV lens (Halle, $f / 2, f=64 \mathrm{~mm}$ ). By means of a beam splitter, a small portion of the incoming laser sheet was directed into a cell containing a diluted solution of fluorescent dye. The generated fluorescence was imaged using a second ICCD camera (LaVision Imager Intense with I/I IRO, $1376 \times 1040$ pixels) equipped with a Nikon lens $(f / 2.8, f=50 \mathrm{~mm})$ and was used for correcting the laser sheet profile inhomogeneities in post processing. The image intensifier was set to an exposure time of



Fig. 2 Schematics of the NO-PLIF experimental set-up.

$400 \mathrm{~ns}$ for both NO-PLIF and dye cell fluorescence cameras. The timing between the different laser pulses and the camera gate openings was synchronized by means of two pulse delay generators (SRS Inc., Model DG532 and BNC, Model 555). A series of 500 single shot measurements was performed at each operating condition. All the images were corrected for background luminosity and also for camera sensitivity. Since there is no off-resonant position between excitation lines for high-pressure conditions, the background luminosity was determined by averaging 500 PLIF single shots without seeding the fuel stream with NO.

\subsection{Mixture Fraction Estimation}

The fuel mixture fraction $(f)$, is defined here as the local mass fraction of all atoms originating from the fuel jet, and can be expressed as,

$$
f=m_{j} /\left(m_{j}+m_{b}\right)
$$

where subscript $j$ denotes the mass originating from the fuel and subscript $b$ denotes the mass from the burned gas stream. $f=1$ corresponds to pure fuel and $f=0$ corresponds to the burned gases. Here it is assumed that the NO seeded to the fuel is not consumed during the mixing process and NO and the fuel are not separated during the mixing process (see also section 3.3.2 for limitations and errors). Therefore, the mass fraction of $\mathrm{NO}\left(Y_{N O}\right)$ within a sample is representative of the fuel mass fraction. $f$ can thus be calculated based on the local NO mass fraction $Y_{N O}$ as [27]

$$
f=\frac{Y_{\mathrm{NO}}-Y_{\mathrm{NO}, \mathrm{b}}}{Y_{\mathrm{NO}, \mathrm{j}}-Y_{\mathrm{NO}, \mathrm{b}}}
$$

Here, $Y_{N O, j}$ is the mass fraction of NO in the pure fuel and the mass fraction of (natural) NO in the burned gas, $Y_{N O, b}$ was below $2 \mathrm{ppm}$ and can be neglected. 
Simple numerical calculations were perfomed to identify the correlations between mixture temperature and the species concentrations. However, the calculations used here do not take into account the temperature and species concentration changes through chemical reactions that can take place during the mixing process, for example, turbulence-chemistry interactions leading to an auto-ignition of the fuel inside the MS. The calculations only mimic the mixing between the exhaust gases and the fuel jet in the MS. The volumetric percentage of each component in the exhaust gas (namely $\mathrm{O}_{2}, \mathrm{~N}_{2}, \mathrm{CO}_{2}$ and $\mathrm{H}_{2} \mathrm{O}$ ) was determined from the probe measurements at the inlet of the MS. The exhaust gas composition in the calculations was simplistically assumed as the equilibrium concentration of a methane/air flame at the global equivalence ratio. Dilution air was added to this mixture to match the exhaust gas $\mathrm{O}_{2}$ concentration from probe measurements. As the fuel jet (which includes the fuel, carrier medium and the tracer) mixes with the hot gases, the mixture composition corresponding to each mixture fraction was calculated. The corresponding mixture temperature was determined by assuming an enthalpy balance between the inflowing reactants (exhaust gases plus the injected fuel mixture) and the end products (fuel/exhaust gas mixture). The specific enthalpies of the hot exhaust gases and the fuel mixture were both calculated from their inflowing enthalpies. Adding these, weighted with their respective mixture fractions $\left(Z_{f u e l}\right.$ for the fuel mixture and $\left(1-Z_{f u e l}\right)$ for the hot exhaust gases), delivered the total enthalpy for a given mixture fraction. This should be equivalent to the enthalpy determined from the calculated mixture composition and temperature for the given mixture fraction. Employing the NASA polynomials, the mixture temperature was iteratively adapted until the enthalpy corresponded to the total enthalpy determined by the inflows, thereby delivering the mixture temperature and species concentrations for a given mixture fraction.

\subsection{Simulation of Theoretical LIF Signals}

The temperature, species concentration and mixture fraction correlations obtained from the simple calculations are used as input parameter for the simulation of theoretical LIF signals using the simulation program LIFSim [28, 29]. The modified LIFSim program evaluates the LIF-signal corresponding to the boundary conditions specified in the input data files. The NO-LIF signal depends not only on the number density of the NO molecules present in the mixture, but also on the pressure, mixture temperature (via the ideal gas law, thermal population of the laser coupled ground state, and the temperature dependent influence of quenching and pressure broadening of absorption lines), and species concentration (via effects like fluorescence quenching). The theoretical LIF signals are calculated using a simple non-transient three-level LIF model that includes equilibrium population of the laser-coupled ground state, a single laser-coupled excited state and fluorescence emission from this single upper state to all possible rotational and vibrational levels of the ground state. The simulation program uses available literature data on the structure of the NO $A^{2} \Sigma^{+}$and $X^{2} \Pi$ states (term energies, transitions strengths) and their LIF properties (collisional line broadening and -shifting, collisional quenching). The program can calculate the absorption, excitation and emission spectra of nitric oxide for a wide range of conditions. State-dependent effects like pressure broadening of absorption lines, thermal population of laser-coupled ground state(s), and quenching of fluorescence by collision of excited NO molecules with other molecules (especially $\mathrm{H}_{2} \mathrm{O}$ and $\mathrm{O}_{2}$ ) are accounted for. The temperature dependence of quenching cross sections has been simulated using the "harpoon" quenching model from Paul et al. [30]. The parameters involved in the model are derived through a fit to experimental quenching data taken from the literatures. Up-to-date spectroscopic data of the NO A-X "gamma" bands and the $\mathrm{O}_{2}$ "Schumann-Runge" bands are used in the program. Further information about the simulation program can be found in [28].

In the linear regime of fluorescence in steady state, the equation for the strength of the LIF signal $I_{L I F}$ can be written as [17]

$$
\begin{aligned}
& I_{L I F}\left(p, T, \widetilde{v}, x_{g a s}\right) \approx f_{B}(T) N_{\mathrm{NO}} I_{v}^{0} \Gamma\left(p, T, \widetilde{v}, x_{\text {gas }}\right) \\
& B \Sigma_{i} A_{i} /\left(A_{i}+Q\left(p, T, x_{g a s}\right)\right)
\end{aligned}
$$

where $f_{\mathrm{B}}$ is the Boltzmann fraction of molecules in the ground state, $N_{\mathrm{NO}}$ the number density of NO molecules, $I_{v}^{0}$ the normalized spectral laser irradiance, $\Gamma$ the spectral overlap of the absorption feature and the laser-line shape, $A$ the rate constant for spontaneous emission, $B$ the Einstein coefficient for stimulated emission, $Q$ the quenching rate, $\widetilde{v}$ the excitation wavenumber and $x_{g a s}$ the gas phase composition. The summation index $i$ here runs over all allowed emission transitions.

The aim of the spectroscopic simulation is to identify the LIF excitation scheme that gives sharp correlation between the LIF signal and the mixture fraction. Experiments are then performed using the selected excitation scheme and the measured 2D NO-LIF images are converted into quantitative, spatially-resolved 2D mixture fraction plots using these correlations.

\subsection{Experimental Challenges}

Quantitative NO-LIF measurements at high pressures are an extremely challenging task, taking into account the various signal interferences that can affect the quality of the measurements. In view of that a detailed assessment of the various sources of errors is necessary. Listed below are some of the problems that were encountered during the current measurements.

2.5.1 Influence of Pressure The LIF signal at high pressures is influenced by three competing processes, (i) an increase in signal intensity with pressure due to an increase in number density, (ii) this increase is counterbalanced by an increase in quenching rate with pressure, and (iii) a decrease in excitation efficiency due to pressure broadening. As a result the NO-LIF emission gets weaker at higher pressures. Moreover, interference from $\mathrm{O}_{2}$ and $\mathrm{CO}_{2}$ LIF emissions increases with pressure due to the molecular line broadening. This is in addition to the signal attenuation by absorption of both the laser 




Fig. 3 Influence of hot $\mathrm{O}_{2}$ on $\mathrm{Q}_{1}(23)$ excitation; Simulations performed using the program LIFSim for $1200 \mathrm{~K}$ and 15 bar for the fuel type F2.

and the fluorescence by hot combustion products like $\mathrm{CO}_{2}$ and $\mathrm{H}_{2} \mathrm{O}$. For the current measurements excitation in the A$\mathrm{X}(0,0)$ band and red-shifted detection is selected based on the relatively high transmission properties of $\mathrm{CO}_{2}$ and $\mathrm{H}_{2} \mathrm{O}$ mentioned in the literature $[28,31]$. Also, to overcome the difficulties associated with the shifting and pressure broadening of NO transitions in the estimation of the excitation line position, an excitation scan was performed before each experiment by uniformly seeding hot cross flow with $\mathrm{NO}$ without any fuel jet, and performing 1D LIF measurements at 15 bar.

2.5.2 Signal Interference One of the main criteria in the selection of NO excitation schemes at high-pressure combustion environments is the transmission properties of the selected line and the LIF interferences from multiple vibrational lines of $\mathrm{O}_{2}$ and broadband $\mathrm{CO}_{2}$-LIF signal (280 $400 \mathrm{~nm})$. For the A-X $(0,0)$ excitation, the $\mathrm{CO}_{2}$ LIF is negligible when compared to the NO signal strengths, but the interfrence from $\mathrm{O}_{2}$ can become decisive depending on the selection of the excitation strategy. The Schumann-Runge bands of $\mathrm{O}_{2}$ overlap with the $\mathrm{NO}$ gamma bands over a wide range of excitation wavelengths resulting in the $\mathrm{O}_{2}$ fluorescence signal overlapping with the NO-LIF signals. Fig. 3 shows the results of the simulation performed using LIFSim for F2 at a temperature and pressure of $1200 \mathrm{~K}$ and $15 \mathrm{bar}$, respectively, showing the $\mathrm{O}_{2}$-LIF interference for the $\mathrm{Q}_{1}(23)$ transition used in the current measurements. For a temperature variation from $1000-1400 \mathrm{~K}$, the simulations showed a 12 fold increase in $\mathrm{O}_{2}$-LIF interference. However, as can be seen from the plot, the interference from $\mathrm{O}_{2}$-LIF is minimal for the selected transition.

2.5.3 Fluorescence Quenching The most dominant quenchers of fluorescence at high temperatures are $\mathrm{O}_{2}$, $\mathrm{CO}_{2}$ and $\mathrm{H}_{2} \mathrm{O}$, whereas $\mathrm{H}_{2}, \mathrm{CH}_{4}$ and $\mathrm{N}_{2}$ are weak quenchers. For example the quenching cross section of $\mathrm{O}_{2}$ at $1249 \mathrm{~K}$ is approximately 68 times greater than that of $\mathrm{N}_{2}$ [32]. For the current investigations in a JICF configuration, the effect of quenching of the NO-LIF signal had a dramatic influence on



Fig. 4 Simulations showing the influence of tracer composition on the fluorescence signal for the excitation line $\mathrm{Q}_{1}(23)$, fuel type F2.

the signal-to-noise $(\mathrm{S} / \mathrm{N})$ ratios, especially for measurements with F2. Initial measurements were performed with the fuel stream seeded with $\mathrm{NO}$ in $\mathrm{N}_{2}(25 / 75 \mathrm{vol}$. \%) as tracer. At regions close to the fuel injection, strong $\mathrm{S} / \mathrm{N}$ was achieved as a result of high number densities of NO molecules and low quenching. But from immediate to far downstream locations where the fuel is mixed with the exhaust gases the $\mathrm{S} / \mathrm{N}$ ratios reduced drastically due to a decrease in number densities as well as due to the effect of quenching. Though all the raw images were corrected for quenching in post processing, the insufficient $\mathrm{S} / \mathrm{N}$ ratios in the raw images made the whole quenching corrections questionable. Also, the dynamic range of the camera used for the LIF detection could not cover the extremely broad intensity range encountered from the jet exit to the downstream region. In order to mitigate this problem, the fuel stream was seeded with $\mathrm{NO}$ in $\mathrm{CO}_{2}(50 / 50$ vol.\%) instead of $\mathrm{NO}$ in $\mathrm{N}_{2}$. The idea behind was on one hand to suppress the dramatic LIF signal reduction by quenching and on the other hand to reduce the dynamic range of the signal due to mixing. Fig. 4 show the variation of simulated theoretical LIF signal $\left(S_{L I F}\right)$ with mixture fraction for the two different cases.

Since NO is mixed in $\mathrm{CO}_{2}$ instead of $\mathrm{N}_{2}$, the NO-LIF signal is already quenched by $\mathrm{CO}_{2}$ within the fuel jet so that the additional signal decrease during mixing by the quenchers in the exhaust gas is not as steep as before.

2.5.4 High Fluorescence Dynamic Range As mentioned above, the high dynamic range of the LIF signals in case of measurements with F2, was a major problem during this investigation. Larger fuel injector diameter implies an increase in volumetric flow rate with a corresponding increase in the signal strength at the jet exit. The problem was mitigated to some extend by using $\mathrm{CO}_{2}$ instead of $\mathrm{N}_{2}$ with $\mathrm{NO}$. However, the difference in $\mathrm{S} / \mathrm{N}$ at the jet exit and downstream locations was still too high. In order to solve this problem a combination of ND filters with a transmission of $50 \%$ and $20 \%$ were used to suppress the NO-LIF signals for measurements at the jet exit. The $\mathrm{S} / \mathrm{N}$ of the single shot raw images varied from a 
maximum of $\approx 48$ at the jet exit to a minimum of $\approx 4$ at the exit of the MS. In comparison, the $\mathrm{S} / \mathrm{N}$ for the raw single shot images with $\mathrm{F} 1$ was $\approx 21$. The raw images (with F2) are then multiplied by the signal attenuation factor of the ND filters in the post processing. One disadvantage of this method was that due to multiplication the noise in the images also gets amplified by the same amount. This resulted in higher noise levels in the images taken at the jet exit when compared to the downstream locations.

2.5.5 Window Staining The high temperatures combined with high pressures and long operating hours at the temperature resulted in staining of the quartz windows with time. The windows became increasingly opaque during operation and this resulted in fluorescence signal loss during the measurements. The influence of this effect on the measurement accuracy is only partly known and difficult to quantify.

\section{Results and Discussion}

\subsection{Correlations Between Mixture Fraction and Theoretical LIF Signal, $S_{L I F}$}

The dependence of the NO-signal/mixture fraction correlation on the NO excitation line is inferred using the spectroscopic simulation program LIFSim (see section 2.4). At high pressures, as complication arises from the line broadening of NO lines, the possibility of finding a single rotational feature of the excitation spectrum is difficult due to spectral overlap. Moreover the aim was to identify excitation schemes that give clear correlations between the NO-LIF signal and the mixture fraction. Fig. 5 shows exemplary excitation schemes and the variation of its normalized $S_{\text {LIF }}$ with mixture fraction for F1 and F2. The simulations were performed for the flow conditions as described in section 2.2.1. The $\mathrm{Q}_{1}(14)$ line has been recommended in the literature $[16,33]$ as optimum for maximum signal strength and minimum interference from $\mathrm{O}_{2}$-LIF at high pressures. However, though the signal strength is relatively weaker than that of $\mathrm{Q}_{1}(14)$, for the current experimental conditions, the excitation scheme $\mathrm{Q}_{1}(23)$ delivered better correlations between $S_{L I F}$ and the mixture fraction. As described in the previous section, for $0.9 \leqslant f \leqslant 1.0$ the sharp decrease in $S_{L I F}$ seen for $\mathrm{F} 2$ is mainly due to quenching. As the fuel mixes with the burned gases $S_{L I F}$ decreases gradually with decreasing NO number densities as witnessed for $0.0 \leqslant f \leqslant 0.9$. For the investigated operating conditions, the stoichiometric $f$ is 0.059 and 0.151 for $\mathrm{F} 1$ and $\mathrm{F} 2$, respectively.

\subsection{Quantitative mixture fraction plots}

NO-PLIF measurements were performed using the optimum LIF-excitation scheme identified using the correlations, $\mathrm{Q}_{1}(23)$. These images were then converted to mixture fraction plots using the respective LIF-signal/mixture fraction correlations for the fuel types F1 and F2, shown in Fig. 5. Fig. 6 shows the mixture fraction plots for the vertical plane at the geometrical axis $(\mathrm{Z}=0 \mathrm{~mm})$ for fuels $\mathrm{F} 1$ and F2. Measurements were also performed at two off-centerline planes, namely $Z=3.5$ and $7 \mathrm{~mm}$. The images shown are from an ensemble average of 500 single shots. Since the height of the



Fig. 5 Examples showing the dependence of NO-LIF signal/mixture fraction with NO excitation schemes. The zoomed region between $0 \leqslant f \leqslant 0.1$ is shown as inset.

laser sheet was only $40 \mathrm{~mm}$, the images at different axial positions are combined to form the mixture fraction plot covering the downstream locations. Also, due to hardware restrictions, it was not possible to image regions close to the jet exit. The region covering up to $2 \mathrm{~mm}$ in the Y-direction for $\mathrm{F} 1$ and $\mathrm{F} 2$, and also up to $5 \mathrm{~mm}$ downstream in the $\mathrm{X}$-direction for F1, was visibly blocked by the frame of the mixing channel window. This resulted in an uncertainity in the measured $f$ values (see section 3.3.1). Fig. 6 shows the development of the mixing process. For the injection of F1, the final mixture fraction distribution is quite symmetric around the centerline $(\mathrm{Y}=0 \mathrm{~mm})$. In contrast, for $\mathrm{F} 2$, the momentum flux ratio is not high enough for the fuel jet to penetrate deep into the cross flow, which would have resulted in better mixing between the jet and the cross flow.

The axial profiles extracted from Fig. 6(a) and (b) are shown in Fig. 7 where the decay of the mixture fraction along the jet centerline $\left(f=f_{\max }\right)$ is plotted against the nondimensionalized jet centerline co-ordinate (s). $s$ is the length of the trajectory from the jet exit, that connects the local maximum or the centerline mixture fraction, $f_{\max }$, at each downstream location, $\mathrm{X}$. The normalization factor $J d$ is suggested in literature as the only global length scale representing the JICF problem [34]. At downstream locations, the plots reveal a faster decay of fuel concentration for F2 than for F1. This is surprising as one would expect faster mixing rate for F1 than for F2, owing to the higher shear rate arising from higher $J$. It will be seen in the following sections that the strong decay of F2 is probably an outcome of the signal loss due to the conversion of seeded $\mathrm{NO}$ to $\mathrm{NO}_{2}$.

In Fig. 8 the radial profiles at different downstream locations for F1, normalized by $f_{\max }$ and the half-width at halfmaximum $\left(r_{h w h m}\right)$ are shown. The profiles shown were taken at different $\mathrm{X}$-locations, vertically across the jet. Here $r$ represents the distance from the jet centerline. The diagnostic method employed is seen to successfully capture the progressive decay from the center value to the shear layer due to rapid entrainment and turbulent mixing. However, the con- 



Fig. 6 2D mixture fraction plots at the geometrical axis $(\mathrm{Z}=0 \mathrm{~mm})$ for the fuel types (a) F1 and (b) F2. Note the different colour bars representing $f$. The black isolines in (a) and (b) correspond to $f=0.05$ and 0.1 , respectively.



Fig. 7 Centerline decay of $f$, deducted from Fig. 6, plotted against the non-dimensionalized centerline co-ordinate $s ; J$ is the momentum flux ratio.

centration profiles in the $\mathrm{Z}=0 \mathrm{~mm}$ plane do not seem to achieve complete self-similarity up to an axial distance of $\mathrm{X} / J d=0.70$. In simple description, complete self-similarity requires the average velocity, scalar concentration and turbulent statistics collapse to a single spatial variable [34]. The small variations seen from $\mathrm{X} / J d=1.14$ onwards is within the accuracy of the measurement technique and the flow seems to achieve self-similarity at downstream locations. There is a strong deviation from the typical Gaussian profile of turbulent jet flows towards the borders, between $r / r_{h w h m}=-1$ to -2 , due to the presence of wake structures.

The gradual development of one part of the counterrotating vortex pair (CVP) with downstream location can be clearly identified in Fig. 9 where the 2D mixture fraction contour plots at different axial locations for the fuel type F2 are shown. The jet column in cross flow transitions into CVP after the jet has turned in the cross flow [34,35]. Due to the afore mentioned problems associated with NO-LIF signal loss, these plots can only be taken as qualitative in nature.



Fig. 8 Radial mixture fraction profiles deducted from Fig. 6(a) normalized by the local maximum value $\left(f_{\max }\right)$ and the half-width at half-maximum $\left(r_{h w h m}\right)$.



Fig. 9 Cross sectional contour plots of $f$ for the fuel type $\mathrm{F} 2$ at different X-locations (a) 10, (b) 30, (c) 50, and (d) $70 \mathrm{~mm}$ showing the growth of one part of the counter rotating vortex pair.

The plots were created by extracting the $1 \mathrm{D}$ plots at different Z-planes $(Z=0,3.5$ and $7.0 \mathrm{~mm})$ and $X$-locations from Fig. 6 and then joined together to form the $2 \mathrm{D}$ cross-sectional plots with interpolation between the Z-planes. It can be seen that the maximum jet concentration is already off-axis or near to the center of the CVP core at an axial distance of $X=10 \mathrm{~mm}$. In general, the growth of the CVP in size with X helps in accelerating the mixing process.

\subsection{Measurement Accuracy}






Fig. 10 Error in $f$ estimation due to an uncertainty in the estimation of NO-LIF intensity corresponding to the calibration at $f=1$. Simulations are for the fuel type F1

\subsubsection{Uncertainty in the estimation of NO-LIF intensity cor-} responding to the calibration at $f=1$ For the quantitative determination of the mixture fraction, the measurement needs a calibration point. This can best be taken within the fuel jet where $f$ is 1 by definition. An uncertainty arises because the immediate exit of jet is blocked visibly by the frame of the mixing channel window. The problem was solved to some extent by ascertaining the maximum intensity, in an arbitrary region close to the fuel exit, from the 500 single shots and use this value as a measure for normalizing (assuming that this value corresponds to $100 \%$ fuel). Doping the cross flow with NO for calibration was hardly feasible due to the high mass flow rate and the complexity of the experimental system. Fig. 10 shows the simulated $S_{\text {LIF }}$ (normalized) vs. $f$ for 3 different cases in which the LIF intensity corresponding to fuel at the jet exit $S_{L I F, \max }$ estimated using the above method is less than the actual value, $S_{L I F, f=1}$. For example, the value corresponds to $f=0.9$ or 0.8 . It can be seen that for a $20 \%$ error in the initial intensity estimation, the maximum error in the mixture fraction estimation is $12 \%$ (corresponding to $S_{L I F}=0.3$ ) for F1. The equivalent maximum error for F2 is $10 \%$ (corresponding to $S_{L I F}=0.14$ ).

3.3.2 Conversion of $\mathrm{NO}$ to $\mathrm{NO}_{2}$ Errors arising from the conversion of seeded $\mathrm{NO}$ to $\mathrm{NO}_{2}$ during measurements turned out to be a major problem in the current investigations. At atmospheric conditions this effect is negligible as the process takes place at a slower rate. But at high pressures and moderate temperatures the reaction rates are different and this effect strongly depends on the local mixture temperature and concentration.

The key reactions involved in this process are shown in Fig. 11 [36-38]. $\mathrm{NO}$ is converted to $\mathrm{NO}_{2}$ by the reaction with $\mathrm{HO}_{2}$. The $\mathrm{NO}$ to $\mathrm{NO}_{2}$ conversion is more efficient for higher $\mathrm{HO}_{2}$-production rates, i.e. for lower temperatures and higher pressures and with respect to the fuel blend, more efficient for $\mathrm{H}_{2}$ rich fuels than for NG like fuels. The reaction scheme shows a NO-oxidation and -reduction cycle in the



Fig. 11 Main reaction pathways in the $\mathrm{NO} / \mathrm{NO}_{2-}$ oxidation/reduction-cycle in a hydrocarbon reaction system with $\mathrm{NO}_{x}$ present ab-initio [38]. In a hydrogen reaction system, the $\mathrm{NO}_{2}$-reduction pathway is missing.

presence of hydrocarbons. In a $\mathrm{H}_{2}$ dominated reaction system, the $\mathrm{NO}_{2}$-reduction pathway via $\mathrm{NO}_{2}+\mathrm{H}$ (dotted line) is not pronounced enough as the effective rate is a function of $[\mathrm{H}]$-atom concentration, which in turn depends on the reaction system progress made towards ignition. Thus, the seeded NO becomes oxidised to $\mathrm{NO}_{2}$, which remains in the system until conditions are changing. Unlike in the hydrocarbon reaction system where the $\mathrm{NO}_{2}$ itself oxidises methyl to methoxy and returns as NO. The short living intermediate methoxy radical itself will decompose to formaldehyde and hydrogen atoms. These hydrogen atoms can form $\mathrm{HO}_{2}$ and $\mathrm{HO}_{2}$ might oxidise $\mathrm{NO}$ again. The $\mathrm{NO}-\mathrm{NO}_{2}$ cycle is very sensitive to the conditions of the $\mathrm{HO}_{2}$ formation. The operating conditions of both measurement series with $\mathrm{H}_{2}$ and $\mathrm{NG}$ as fuels at temperatures ranging from $1000-1300 \mathrm{~K}$ at a pressure of 15 bar are very favourable for $\mathrm{HO}_{2}$ formation. However, the $\mathrm{NO}$ to $\mathrm{NO}_{2}$ conversion will be less for measurements at lower pressures (for example, at $p=5$ or $10 \mathrm{bar}$ ) as the effect is less pronounced under those conditions.

Kinetic simulations were performed to ascertain the implications of this effect on the current measurements. The input boundary conditions for the simulations were derived from the experimental operating conditions. The exhaust gas mixture composition was modelled with a PSR/PFR-sequence (Perfectly Stirred Reactor/Plug Flow Reactor) simulating a natural gas combustion at lean conditions and cooling air admixture. This resulted in the modelled exhaust gas composition reproducing the experimentally determined exhaust gas composition at the inlet of the MS. The reaction system was then initialised with the admixture of the fuel/tracer blend to the exhaust gas using PSR/PFR-sequence. The calculations of the kinetic system used the RDv06-NGQ reaction mechanism for $\mathrm{NG}$, extended with $\mathrm{NO}_{x}$-kinetics, and was validated in [38-40]. The simulations were then repeated for conditions richer and leaner than the experimental operating conditions, $X / X_{\text {ref }}=1$ (reference conditions in mole fraction, where $\phi_{\text {global }}$ inside the MS are 0.47 and 0.36 for F1 and F2 respectively). This was done to imitate the mechanisms taking place at regions close to the core of the fuel jet, where the mixture is relatively rich and cold, and at the shear layer of the jet, where the mixture is lean and hot. The temperature of the exhaust gases were fixed at the reference conditions. The results of these simulations are shown in Fig. 12 where the decrease in [NO] in percentage and $T_{m i x} / T_{r e f}$ at $t=0.5 \mathrm{~ms}$ 




Fig. 12 Variations in the NO concentration and mixture temperature $0.5 \mathrm{~ms}$ after fuel injection into the MS; Stars: fuel type F1, Circles: fuel type F2. $X / X_{\text {ref }}=1.0$ represents the $f_{\text {global }}$, which is 0.029 for $\mathrm{F} 1$ and 0.06 for $\mathrm{F} 2$. $\mathrm{X} / \mathrm{X}_{\text {ref }}=2.0$ is equivalent to $f=0.05$ and 0.1 for $F 1$ and $F 2$ respectively.

after fuel injection are shown with respect to the variations in the fuel jet concentration $\left(X / X_{\text {ref }}\right)$. Here, $0.5 \mathrm{~ms}$ represents the minimum residence time of the fuel molecule $(t=0$ at the jet exit) inside the MS shown in Fig. 6 and $T_{m i x}, T_{r e f}$ are the mixture temperature after $0.5 \mathrm{~ms}$ and the cross flow temperature, respectively. At this point it needs to be stressed that though the progress of the reactions of the major species is important for the NO-NO 2 cycle, the variations in the major species concentration and the mixture temperature was found to be negligible. So, errors arising from the effect of reaction on the calculation of the correlation between NO-LIF signal (with respect to temperature and quenching rate) and mixture fraction are assumed to be minor for the experimental conditions in this work.

As the fuel is injected, $T_{m i x}$ decreases from the reference value and the effect is stronger with increased fuel concentrations. With changes in fuel concentration, the decrease in [NO] for F1 varies from $38 \%$, for very lean conditions, to $23 \%$, for relatively rich conditions, in $0.5 \mathrm{~ms}$. The $\mathrm{NO}_{2}$ produced is rapidly converted back to NO by the methyl radical. In comparison, the fluctuation is severe for F2, varying from a maximum of $88 \%$ to a minimum of $58 \%$. The initial fall in $[\mathrm{NO}]$ decrease from $X / X_{\text {ref }}=0.1$ to 0.4 due to the rapid reconversion from $\mathrm{NO}_{2}$ to $\mathrm{NO}$ via $\mathrm{NO}_{2}+\mathrm{H}$. This rests on the higher $[\mathrm{H}]$-atom concentrations because the reaction system at $t=0.5 \mathrm{~ms}\left(<\approx \tau_{\text {ign }}\right.$, the ignition delay time $)$ already approaches ignition because of higher initial mixing temperatures for lower $X / X_{\text {ref }}$. Vice versa, the turnaround for increasing $X / X_{r e f}$ is caused by the decreasing initial $T_{m i x}$ and the increasing $\tau_{i g n}$. Thus the $[\mathrm{H}]$-atom concentrations recorded at $t=0.5 \mathrm{~ms}$ ( $\ll \tau_{\text {ign }}$ ) are much lower compared to the $\left[\mathrm{HO}_{2}\right]$-radical concentrations and therefore the effective



Fig. 13 PSR calculations showing the temporal variation of [NO] normalized by their start values; Closed symbols: fuel type F1, Open symbols: fuel type F2

rate of $\mathrm{NO}_{2}+\mathrm{H}$, i.e. the $\mathrm{NO}_{2}$-reduction pathway, is slowed down drastically. Since the plot shown includes the effect of both fuel concentration and mixture temperature variations, the simulations were performed again (not shown) for a constant fuel concentration and varying exhaust gas temperatures, which can highlight the influence of $T_{\text {mix }}$ on the plots shown in Fig. 12. The simulations showed that the conversion of $\mathrm{NO}$ to $\mathrm{NO}_{2}$ via $\mathrm{HO}_{2}$ is indeed temperature sensitive for both cases, in the sense that the forward reaction leading to the production of $\mathrm{HO}_{2}$ is accelerated only when a favourable temperature is attained. This further explains the downward slope of [NO] decrease from $X / X_{\text {ref }}=1.5$ onwards.

For the current measurements the above results have the following implications. At the core of the fuel jet where the mixture is rich and the mixture temperatures are low, there is only minor conversion of $\mathrm{NO}$ to $\mathrm{NO}_{2}$. For example, $X / X_{\text {ref }} \geqslant 2.0$ the $[\mathrm{NO}]$ decrease is less than 23 and $83 \%$ for F1 and F2, respectively. From homogeneous mixing calculations without reaction, $X / X_{r e f} \geqslant 2.0$ is equivalent to $f \geqslant 0.05$ and 0.1 for the fuels F1 and F2. This implies that for $\mathrm{F} 1$, the reported $f$ values have a maximum error between 23 to $38 \%$ for $0 \leqslant f \leqslant 0.05\left(0 \leqslant X / X_{\text {ref }} \leqslant 2.0\right)$ and the measurement accuracy is within $\pm 12 \%$ for $f \geqslant 0.05$. In case of F2, at regions close to the shear layer near to the jet exit, and at downstream locations, where the mixture temperatures are favourable for $\mathrm{NO}$ to $\mathrm{NO}_{2}$ conversion, the $\mathrm{NO}$ signal reduction and thereby the error in the mixture fraction estimation seems to be appreciable. Fig. 13 shows the temporal variation of $[\mathrm{NO}]$ for $X / X_{r e f}=0.5,1.0,1.5$, and 2.0 for the two fuels. The graph reveals a non-linear relationship between [NO] reduction and $t$ for the fuel F2. It also highlights the reconversion of $\mathrm{NO}_{2}$ to $\mathrm{NO}$ with $t$ for $X / X_{r e f}=0.5$. This implies that at shear layers or at downstream locations, where $0 \leqslant f \leqslant 0.1$, the reported $f$ values becomes rapidly unreliable. At these regions there is a spatial variation in the accuracy of reported values in Fig. 6 depending on the local $f, T_{\text {mix }}$, and $t$. In view of this, the $f$ values reported here for F2 can be considered only as semi-quantitative in nature. The 
isolines representing $f=0.05$ and 0.1 are shown as an overlay in Fig. 6(a) and (b), respectively.

3.3.3 Signal loss due to window staining As mentioned before in section 2.5 one of the problems that was encountered during these measurements was the change in window transmission properties due to staining of the windows from long operating hours and high temperatures. This resulted in a decrease in the laser energy as well as the LIF signal transmission with time. The transmission loss was approximately $2 \%$ during the period of measurements. While the corrections for the shot-to-shot variations in laser energy have been accounted for in the post processing phase, corrections for the signal loss is not possible as it is a function of the spatial coordinates. In effect this resulted in the reported $f$ values being underestimated when compared to the real values.

3.3.4 Unwanted signals The different unwanted signals that are present in the measured signal are the dark signal - the fixed pattern signal that occurs without any incident fluorescence on the ICCD and the background signal - the signal resulting from laser reflections, natural flow luminosity etc. In the current measurements the background signal is reduced to a great extent by gating, by using special filters and also by subtracting a background image, taken without NO seeding, from the measured images. The error in $f$ estimation arising from the dark signal was found to be approximately $4 \%$.

\section{Summary and Conclusions}

Fuel concentration and mixture fraction measurements of the fuel using LIF of NO as the trace species at high pressures and high temperatures are reported in this paper. Two different fuels, designated as F1 and F2, with F1 consisting of natural gas (NG) and $\mathrm{F} 2$ of $\mathrm{NG}, \mathrm{H}_{2}$, and $\mathrm{N}_{2}$, were investigated. The measurements were performed in jet in cross flow (JICF) configuration in which the fuel together with the trace species was injected into a cross flow of hot exhaust gases. Since the NO-LIF signal has a non-linear relationship with the mixture fraction, numerical calculations that describe the thermochemical state of the experimental scenario were used to support the spectroscopic simulation of the theoretical LIF signal. These simulations were used to identify the optimum excitation scheme that delivered sharp correlations between the mixture fraction and the LIF signal. The $\mathrm{Q}_{1}(23)$ rotational line of the NO-LIF spectrum, which showed good correlations was used for the current experimental configuration. The measured NO-LIF images were then converted into mixture fraction plots using the simulated correlations.

In spite of the various challenges encountered during the measurements, the diagnostic method employed was successful in capturing the dynamics of the JICF configuration. The measurements showed that the F2 fuel jet $\left(\mathrm{NG}, \mathrm{H}_{2}\right.$, and $\mathrm{N}_{2}$ ) slightly underpenetrates in comparison to $\mathrm{F} 1 \mathrm{NG}$ ). This in turn affected the mixing rate between the two streams resulting in a non-uniform fuel/oxidizer mixture at the exit of the mixing section. The radial mixture fraction profiles at different axial locations showed the progressive concentration decay from the center value to the shear layer as a result of rapid entrainment of the burned gases and turbulent mixing. A slight non-similarity in the mixture fraction profiles was seen at regions close to the jet exit which became more similar at downstream locations. By extrapolating the 1D profiles at different planes parallel to the geometrical axis it was possible to visualize the growth of the counter rotating vortex pair and its influence on the fuel/burned gas mixing with axial distance.

In addition to the known difficulties associated with concentration measurements using LIF at high pressures like pressure broadening, fluorescence quenching, signal interference from molecules like $\mathrm{O}_{2}$ and $\mathrm{CO}_{2}$ - LIF, the measurements encountered additional difficulties in the form of signal loss due to window staining and conversion of seeded $\mathrm{NO}$ into $\mathrm{NO}_{2}$, which was detrimental to the accuracy of the measured values. The problem was more severe for the $\mathrm{H}_{2}$ dominated fuel F2 than for F1. The kinetic simulations on the basis of a well-stirred reactor model revealed the complex dependence of the NO- $\mathrm{NO}_{2}$ conversion on temperature and mixture composition and the dominant role of $\mathrm{HO}_{2}$ in this process. Unfortunately, the conditions prevailing in the experiment were very favourable for the formation of $\mathrm{HO}_{2}$. While the measurements suffered a spatial variation in accuracy due to the $\mathrm{NO}-\mathrm{NO}_{2}$ conversion, the errors inflicted by the staining of the optical windows were not clearly quantifiable. Therefore the mixture fraction values reported in this paper for F2 are more semi-quantitative in nature. However, in the future measurements it should be possible to alleviate the error arising from the NO- $\mathrm{NO}_{2}$ conversion by including the species concentration and temperature changes due to reaction in the estimation of the thermochemical state of the experimental conditions. The spectroscopical simulation of the LIF signalmixture fraction correlation using these thermochemical data will then intrinsically include the effects of $\mathrm{NO}-\mathrm{NO}_{2}$ conversion on the LIF signal.

Acknowledgements The work has been funded by ALSTOM Power Generation AG, EnBW Holdging AG and the "Ministerium für Wissenschaft, Forschung und Kunst (MWK) Baden Württemberg", which is greatfully acknowledged. The authors are greatful to Dr. Wolfgang Bessler for providing access to the LIFSim code. The authors would also like to acknowledge the technical support of $U$. Prestel, K.H. Ferst and S. Peuckert.

\section{References}

1. J. Wolfrum, Proc. Combust. Inst., 27 (1998) 1-41.

2. K. Kohse-Höinghaus, J. Jeffries (Eds.), Applied Combustion Diagnostics (Taylor \& Francis, New York 2002).

3. C. Orlemann, C. Schulz, J. Wolfrum, Chem. Phys. Lett., 307 (1999) 15-20.

4. J. Fielding, J.H. Frank, S.A. Kaiser, M.D. Smooke, M.B. Long, Proc. Combust. Inst., 29 (2002) 2703-2709.

5. J. Kazenwadel, W. Koban, T. Kunzelmann, C. Schulz, Chem. Phys. Lett., 345 (2001) 259-264.

6. R. Barlow, Proc. Combust. Inst., 31 (2007) 49-75.

7. W. Meier, P. Weigand, X.R. Duan, R. Giezendanner-Thoben, Combust. Flame, 150 (2007) 2-26.

8. J.A. Sutton, J.F. Driscoll, Exp. Fluids, 41 (2006) 603-627. 
9. J.A. Sutton, J.F. Driscoll, Proc. Combust. Inst., 31 (2007) 14871495 .

10. T.J. McIntyre, H. Kleine, A.F.P. Houwing, Aeronaut. J., 111(1115) (2007) 1-16.

11. T. Rossmann, M.G. Mungal, R.K. Hanson, Appl. Opt., 42(33) (2003) 6682-6695.

12. C. Schulz, V. Sick, Prog. Energy Combust. Sci., 31 (2005) 75121.

13. A.O. Vyrodov, J. Heinze, U.E. Meier, J. Quant. Spectrosc. Radiat. Transfer, 53(3) (1995) 277-287.

14. J.R. Reisel, N.M. Laurendeau, Combust. Sci. Technol., 98 (1994) 137-160.

15. T. Lee, W. Bessler, H. Kronemayer, C. Schulz, J.B. Jeffries, Appl. Opt., 44(31) (2005) 6718-6728.

16. M. Di Rosa, K.G. Klavuhn, R.K. Hanson, Combust. Sci. Technol., 118 (1996) 257-283.

17. A.C. Eckbreth, Laser Diagnostic for Combustion Temperature and Species (Gordon and Breach, Netherlands 1996).

18. R. Sadanandan, R. Schiessl, D. Markus, U. Maas, Flow Turbul. Combust., 86(1) (2011) 45-62.

19. R. Schiessl, S.A. Kaiser, U. Maas, M.B. Long, Proc. Combust. Inst., 32 (2009) 887-894.

20. A.R. Karagozian, Prog. Energy Combust. Sci., 36 (2010) 531553.

21. J.U. Schlüter, T. Schönfeld, Flow Turbul. Combust., 65 (2000) 177-203.

22. J. Fleck, P. Griebel, A.M. Steinberg, M. Stöhr, M. Aigner, A. Ciani, Proc. of ASME Turbo Expo, GT2011-46195 (2011) (accepted).

23. F. Güthe, J. Hellat, P. Flohr, J. Eng. Gas Turb. Power, 131 (2009) 021503 1-7.

24. J. Fleck, P. Griebel, A.M. Steinberg, M. Stöhr, M. Aigner, A. Ciani, Proc. of ASME Turbo Expo, GT2010-22722 (2010).

25. R. Lückerath, W. Meier, M. Aigner, J. Eng. Gas Turb. Power, 130 (2008) 011505 1-7.

26. M. Namazian, J. Kelly, R. Schefer, Proc. Combust. Inst., 25 (1994) 1149-1157.

27. J. Warnatz, U. Maas, R.W. Dibble, Combustion (Springer, Berlin 2006).

28. W. Bessler, PhD. thesis, University of Heidelberg (2003).

29. W. Bessler, C. Schulz, V. Sick, J. Daily, Proceedings of the Third Joint Meeting of the U.S. sections of the Combustion Institute, D33 (2003) 1-6.

30. P.H. Paul, C.D. Carter, J.A. Gray, J.L. Durant Jr., J.W. Thoman Jr., Appl. Phys. B, 57 (1993) 249-259.

31. C. Schulz, J.D. Koch, D.F. Davidson, J.B. Jeffries, R.K. Hanson, Chem. Phys. Lett., 355 (2002) 82-88.

32. T.B. Settersten, B.D. Patterson, J. Chem. Phys., 124 (2006) 234308 1-14.

33. W. Bessler, C. Schulz, J.D. T. Lee, J.B. Jeffries, R.K. Hanson, Appl. Opt., 41(18) (2002) 3547-3557.

34. S.H. Smith and M.G. Mungal, J. Fluid Mech., 357 (1998) 83122.

35. T.F. Fric, A. Roshko, J. Fluid Mech., 279 (1994) 1-47.

36. P. Dagaut, A. Nicolle, Combust. Flame, 140 (2005) 161-171.

37. T. Faravelli, A. Frassoldati, E. Ranzi, Combust. Flame, 132 (2003) 188-207.

38. J. Herzler, C. Naumann, 23rd ICDERS, Irvine, USA (2011) (submitted).

39. J. Herzler, C. Naumann, Proc. Combust. Inst., 32 (2009) 213220.

40. G. Weiss, W. Meier, Abschlussbericht Forschungsinitiative "Kraftwerke des 21. Jahrhunderts (KW21)", (2009) 619-638. 